\title{
ABELIAN SELF-COMMUTATORS IN FINITE FACTORS
}

\author{
GABRIEL NAGY
}

\begin{abstract}
An abelian self-commutator in a $\mathrm{C}^{*}$-algebra $\mathcal{A}$ is an element of the form $A=$ $X^{*} X-X X^{*}$, with $X \in \mathcal{A}$, such that $X^{*} X$ and $X X^{*}$ commute. It is shown that, given a finite $\mathrm{AW}^{*}$-factor $\mathcal{A}$, there exists another finite $\mathrm{AW}^{*}$-factor $\mathcal{M}$ of same type as $\mathcal{A}$, that contains $\mathcal{A}$ as an $\mathrm{AW}^{*}$-subfactor, such that any self-adjoint element $X \in \mathcal{M}$ of quasitrace zero is an abelian self-commutator in $\mathcal{M}$.
\end{abstract}

Mathematics subject classification (2000): 46L35, 46L05.

Key words and phrases: Self-commutator, AW*-algebras, quasitrace.

\section{REFERENCES}

[1] S. BERBERIAN, Baer *-rings, Springer-Verlag, New York-Heidelberg-Berlin, 1972

[2] B. Blackadar, D. Handelman, Dimension functions and traces on $C^{*}$-algebras, J. Funct. Analysis. Vol. 45, 1982

[3] U. HaAgerup, Quasitraces on exact $C^{*}$-algebras are traces, Preprint 1991

[4] I. KAPlansky, Rings of Operators, W. A. Benjamin Inc. New York, 1968

[5] S.H. KIM, G. NAGY, Spectral symmetry in $\mathrm{II}_{1}$-factors, Preprint 2004, arXiv.org: math.OA/0408275

[6] F.J. Murray, J. von NeumanN, On Rings of Operators, Ann. Math. Vol. 37, No. 1, 1937 